Spin Spectrometer at the ALS and APS-NIM/SRI07

\title{
Spin Spectrometer at the ALS and APS
}

\author{
J.G. Tobin ${ }^{1}$, S.A. Morton ${ }^{1,2, *}$, S.W. Yu ${ }^{1}$, T. Komesu' ${ }^{2}$, G.D. Waddill' ${ }^{2}$ and P. Boyd ${ }^{3}$
}

\author{
1.Lawrence Livermore National Laboratory, Livermore, CA \\ 2.University of Missouri-Rolla, Rolla, $\mathrm{MO}$ \\ 3.Boyd Technologies, Santa Rosa, CA \\ *Present Location: Lawrence Berkeley Laboratory, Berkeley, CA
}

Abstract

A spin-resolving photoelectron spectrometer, the "Spin Spectrometer," has been designed and built. It has been utilized at both the Advanced Light Source in Berkeley, CA, and the Advanced Photon Source in Argonne, IL.

Technical details and an example of experimental results are presented here.

Discussion

Spin resolved photoemission is a powerful technique that includes both energy and spin analysis of the collected photoelectrons. Using the "Spin Spectrometer" (Figures 1-3), our group has used this technique to investigate a number of systems [1-10], at both the Advanced Light Source [1-6] and the Advanced Photon Source [7-10].

The key measurements are based upon spin-resolving and photondichroic photoelectron spectroscopy. True spin-resolution is achieved by the use of a Mini-Mott detection scheme. The photon-dichroic measurements include the variant magnetic $x$-ray linear dichroism (MXLD). Both a multi-channel, energy dispersive collection scheme as well as the spin-detecting Mini-Mott apparatus are used in data collection. The "Spin Spectrometer" was originally based at the Spectromicroscopy Facility (Beamline 7) at the Advanced Light Source, using linearly polarized $x$-rays, and subsequently moved to the circularly polarization facility at Beam-line 4 at the Advanced Photon Source. The high angular and energy resolution with high throughput is achieved via the use of an 11-inch mean diameter hemispherical analyzer supplied by Physical Electronics. (Figure 1) Included in this package is an electron collections lens stack with an 
adjustable aperture, permitting selection of various angular and sample spot sizes. The novel aspect of our $\mathrm{PHI}$ analyzer is that the multi-channel detector has a hole in the center, permitting the direct passage of energy analyzed electrons into the electron optics without resorting to an electron switch yard. [6] The presence of the hole does cause some problems when the multi-channel (non-spin) detection is being used: an increase in dark and background counts. Dark counts are defined as non-zero electron counting that occurs when the multi-channel detection is "on" but no excitation is striking the sample. Background counts are the counts underlying the elastic photoelectron peaks, e.g., a core-level, when actual collection is underway. Regardless, under many conditions, these problems are inconsequential. Spin resolution is achieved by directing the electrons through the optics and into the Mini-Mott detector. In this case, the high voltages on the channel plates are turned off and the channel plates and anode assembly become part of the first lens stack, directing the electrons into the $90^{\circ}$ spherical sector. The $90^{\circ}$ sector is run at a relatively high pass energy: energy resolution is provided solely by the hemisphere and the photon monochromator. Because the multi-channel detection is at the exit plane of the hemisphere, the imaging of the hemisphere entrance slit onto the multichannel analyzer is unperturbed and high resolution non-spin counting is achieved. Furthermore, since the spin resolving detection does not require precise imaging, the burden of high resolution spatial imaging is lifted from the $90^{\circ}$ sectors. This allows the $90^{\circ}$ sectors to be run at high pass energies, optimizing throughput. The $90^{\circ}$ sector serves one salient purpose: it allows the simultaneous resolution of both the vertical spin (Px) and the spin along the electron emission direction(Pz). (Figure 2) These two components are of particular importance in our experiments. After the $90^{\circ}$ sector, the electrons travel through another lens stack, into the Mini-Mott detector. In the Mini-Mott, the electrons are accelerated to $24 \mathrm{kV}$, with four channeltrons positioned horizontally and vertically used for electron counting. While suffering from a relatively low figure of merit, the Mini-Mott has two key advantages to our design. First, it provides essentially turn-key operation with the requirement of no special 
Spin Spectrometer at the ALS and APS-NIM/SRI07

preparation. Second, it has been shown that the electron optical matching of a spin detector to a large hemisphere can be optimized by the use of a Mini-Mott.

Finally, an example of our spin resolved data is shown in Figure 3. [11] Note the sharpness of the Fermi Edge and the strong spin-specific nature of individual spectral structures.

\section{Acknowledgements}

This work was performed under the auspices of the U.S. Department of Energy, by the University of California Lawrence Livermore National Laboratory under contract W-7405-Eng-48. Work that was performed by LLNL and UMR personnel was supported by the Office of Basic Energy Science at the U.S Department of Energy. The ALS and the APS have been built and operated under funding from the Office of Basic Energy Science at DOE.

\section{References}

1. M. Hochstrasser, J.G. Tobin, E. Rotenberg and S.D. Kevan, Phys. Rev. Lett. 89, 216802 (2002).

2. F.O. Schumann and J.G. Tobin, Surf. Sci. Lett., 476, L235 (2001).

3. J.G. Tobin and F.O. Schumann, Surf. Sci., 478,211 (2001).

4. S.A. Morton, G.D. Waddill, S. Kim, I.K. Schuller, S.A. Chambers, and J.G. Tobin, Surface Science Letters 513, L451 (2002).

5. J.G. Tobin, S.A. Morton, S.W. Yu, G.D. Waddill, I.K. Schuller, and S.A. Chambers, J. Phys. Cond. Matter 19, xxxx (2007)

6. J.G. Tobin P.J. Bedrossian, T.R. Cummins, G.D. Waddill, S. Mishra, P. Larson, R. Negri, M. Miller, E. Peterson, P. Boyd and R.F. Gunion, "Preliminary Results from a New Spin Spectrometer," MRS Symp. Proc. 524, 185 (1998).

7. S.W. Yu, T. Komesu, B.W. Chung, G.D. Waddill, S.A. Morton, and J.G. Tobin, Phys. Rev. B 73, 075116 (2006).

8. Takashi Komesu, G.D. Waddill, and J.G. Tobin, J. Phys.: Cond. Matt. 18,8829(2006).

9. J.G. Tobin, S.W. Yu, T. Komesu, B.W. Chung, and G.D. Waddill, EuroPhys Lett. (EPL) 77, 17004 (2007).

10.T. Komesu, G.D. Waddill, and J.G. Tobin, Phys. Lett.A, in press, 2007

11.M. Spangenberg, J.R. Neal, T.H. Shen, S.A. Morton, J.G. Tobin, G.D. Waddill, J.A.D. Matthew, D. Greig, A.E.R. Malins, E.A. Seddon, M. Hopkinson, J. Mag. Mag. Matl. 292, 241 (April 2005).

Nuclear Instrumentation and Methods 


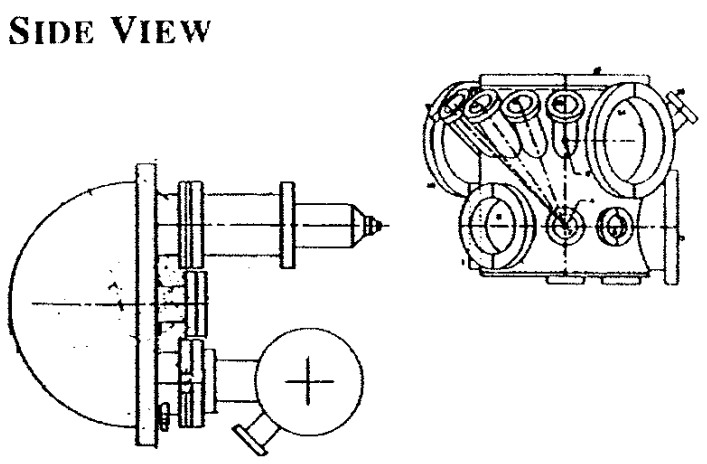

TOP VIEW
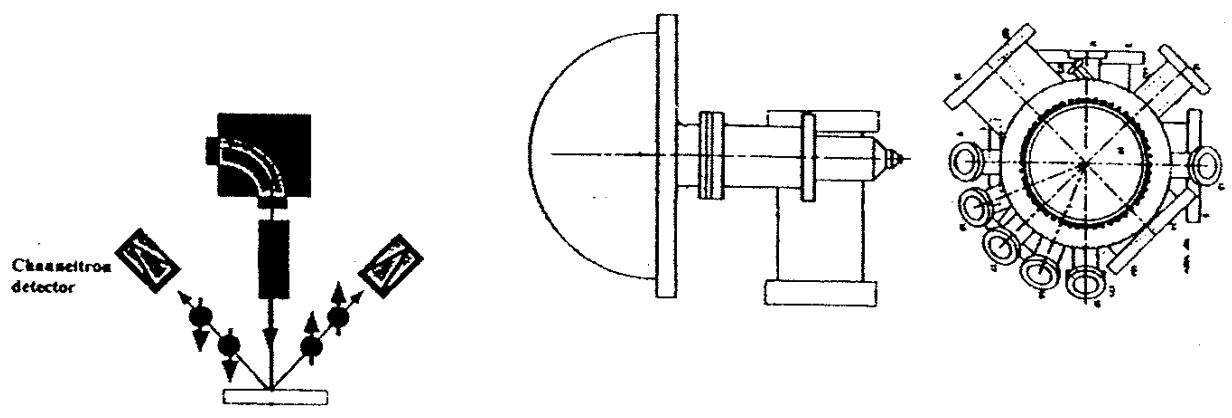

Figure 1

Top and side views of the energy analyzer and vacuum vessel are shown here. The ports include access for LEED, magnetizing coils, evaporation, and sample manipulation. The analyzer has a mean radius of 5.5 inches. The images of the analyzer and vacuum vessel are scaled identically here. The analyzer has an acceptance lens system on the top and the lens stack, 90 degree sector and MiniMott on the bottom. M. Hochstrasser has provided part of this figure. 


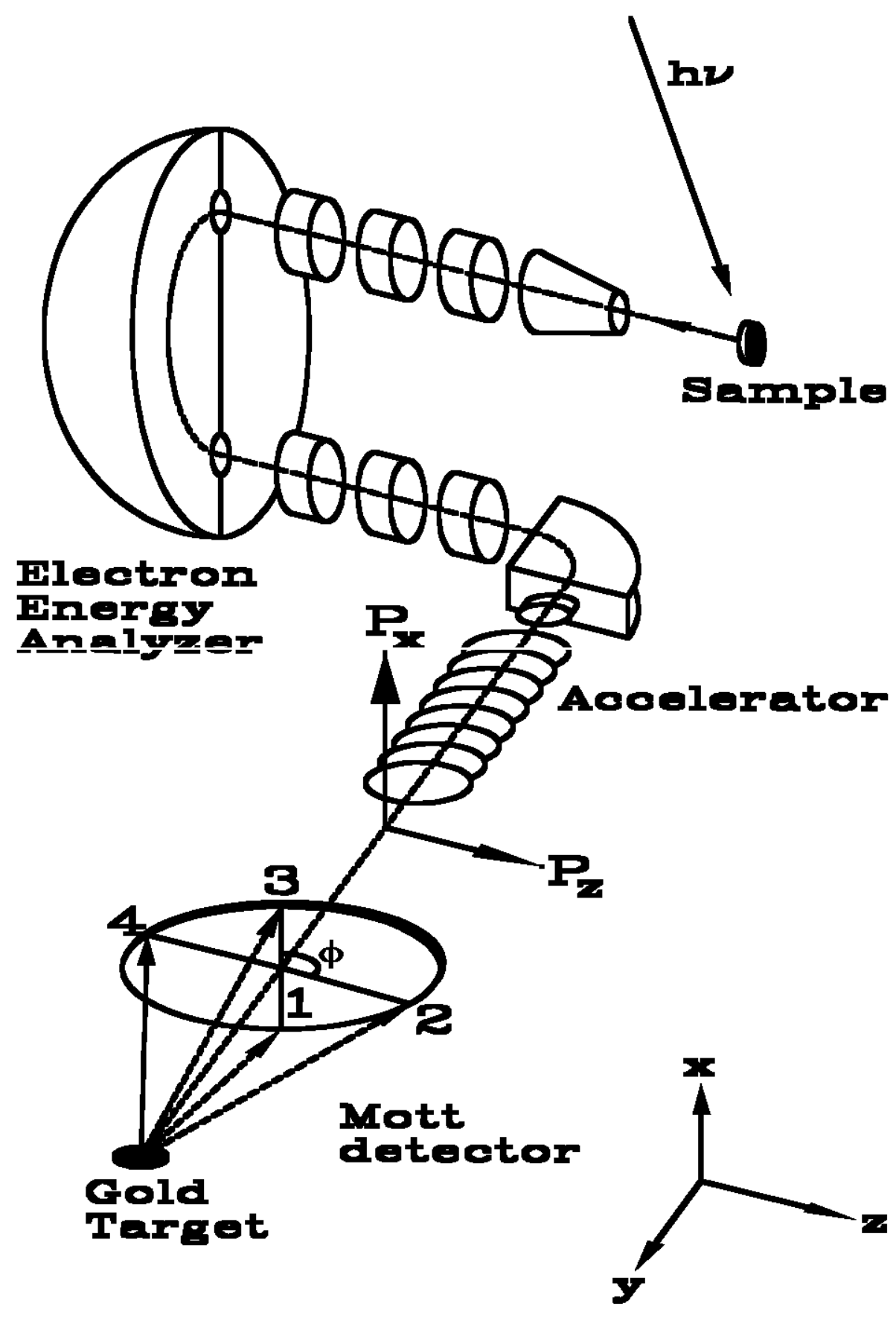

Figure 2

A schematic diagram of the electron analyzer is shown here, including various geometrical relationships.

Nuclear Instrumentation and Methods

Synchrotron Radiation Instrumentation Conference, 2007 


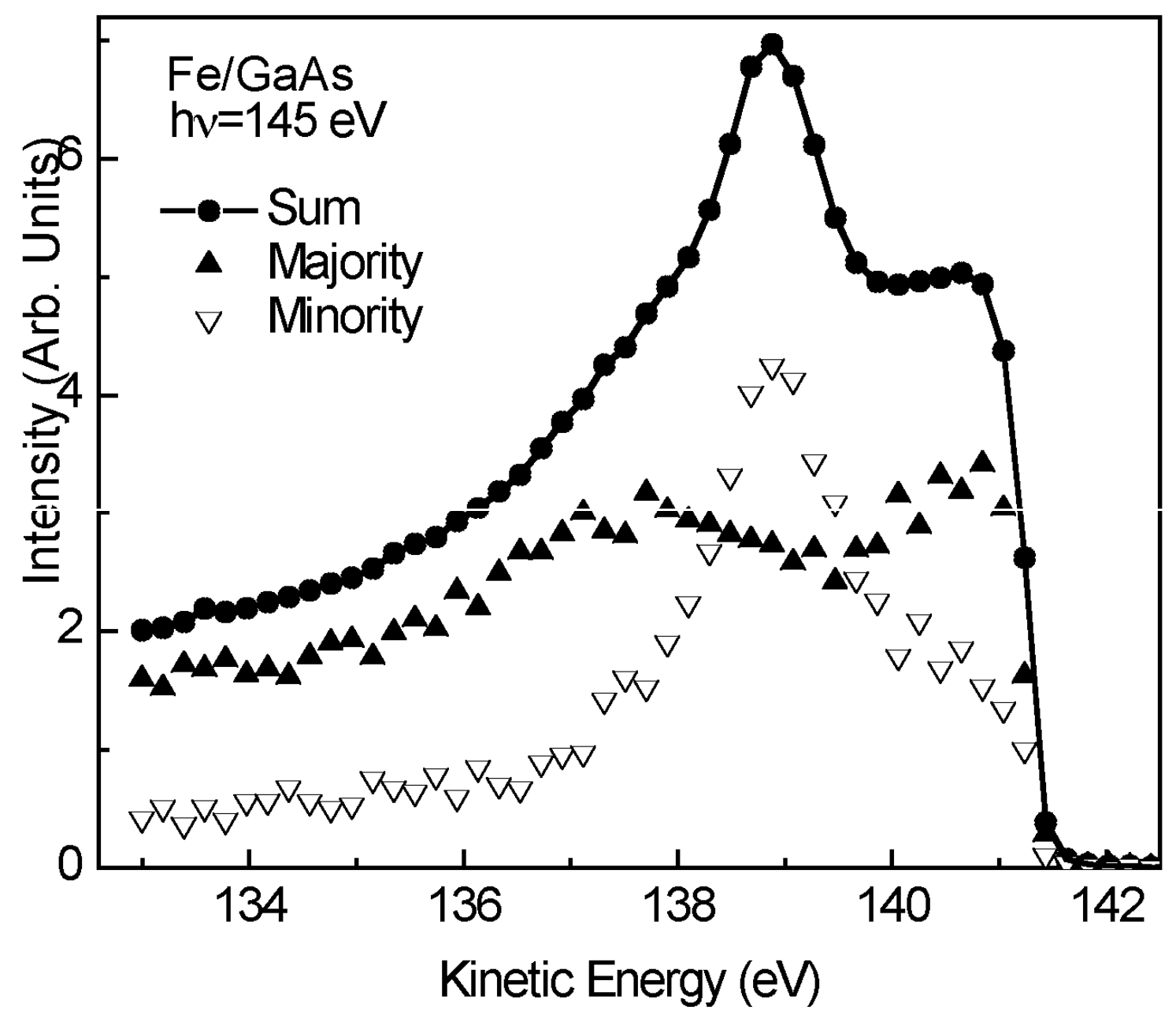

Figure 3

An example of some data collected on Beamline 7 at the ALS is shown here. The photon energy was $145 \mathrm{eV}$ and the photon polarization is linear. The spinresolved valence band photoemission results are from Fe overlayers, as part of a larger collaboration. [11] The Fermi edge is near 141 eV Kinetic Energy. 